\title{
FACTORES ASOCIADOS A LA APENDICITIS AGUDA COMPLICADA EN UN HOSPITAL PÚBLICO DE LA CIUDAD DE ICA, OCTUBRE 2018 - SETIEMBRE 2019
}

\author{
Factors associated with complicated acute appendicitis in a public hospital in the City of Ica, \\ October 2018 - September 2019 \\ Isabel Natividad Urure Velazco1,2,a,d, Luisa Antonia Pacheco Villa García $3, a, c$, Karen Leticia Llerena Ururi4,b, \\ Ana María Cisneros Quispe ${ }^{2, a}$
}

\begin{abstract}
${ }^{1}$ Facultad de Enfermería de la Universidad Nacional San Luis Gonzaqa de Ica, Perú, ${ }^{2}$ Hospital Santa María del Socorro de Ica, Perú, ${ }^{3}$ Hospital Regional de Ica, Perú, ${ }^{4}$ Instituto Nacional de salud del Niño de Lima, Perú, a Licenciada en enfermería ${ }^{b}$ Médico cirujano, ${ }^{c}$ Magíster en Salud Pública, ${ }^{d}$ Doctor en ciencias de la salud.
\end{abstract}

\section{RESUMEN}

La apendicitis aguda es el cuadro quirúrgico de urgencia más frecuente, y es la causa principal de abdomen agudo quirúrgico. Objetivo: Identificar los factores asociados a la apendicitis aguda complicada en un hospital público de la ciudad de Ica. Material y Métodos: Estudio descriptivo, observacional, analítico, prospectivo, no experimental. Muestra: Constituida por 147 pacientes mayores de 14 años postoperados de apendicitis aguda, obtenidos de diciembre 2018 a julio 2019, utilizándose como base, formularios y/o fichas validados a nivel internacional y adaptado a nuestra realidad, para recojo de información de la historia clínica del paciente, y una encuesta con 3 reactivos de respuestas dicotómicas. Resultados: Predominó el sexo masculino 56,5\% y el grupo etario de 20 a 29 años 32,7\%. Según la clasificación clínica: Mostró apendicitis aguda complicada 53,1\% (78 pacientes) y apendicitis aguda no complicada 46,9\% (69 pacientes). Referente a estadios histopatológicos fue flemonosa o supurada 33,3\%, gangrenosa o necrótica y perforada con $26,5 \%$ ambos, y congestiva o catarral $13,6 \%$. El tiempo transcurrido entre el inicio de los síntomas y el ingreso al hospital fue de menos de 24 horas 51,7\%. Tiempo transcurrido entre el ingreso al hospital y la intervención quirúrgica reportó más de 6 horas con $68 \%$. La automedicación fue 65,3\%, no recibieron atención médica pre hospitalaria 59,9\%. En las complicaciones intraabdominales predomina la peritonitis localizada y plastrón apendicular con $50 \%$ y $12,5 \%$ respectivamente. Conclusiones: La apendicitis aguda complicada se asoció a factores como la automedicación y el tiempo transcurrido entre el inicio de los síntomas y la intervención quirúrgica.

Palabras clave: Apendicitis aguda complicada y factores asociados.

\section{SUMMARY}

The appendicitis acute is the most frequent emergency surgical condition, and is the leading cause of acute surgical abdomen. Objective: To identify the factors associated with complicated acute appendicitis in a public hospital in the city of Ica. Material and Methods: Descriptive, observational, analytical, prospective, non-experimental study. Sample: Made up of 147 patients over 14 years of age after acute appendicitis, obtained from December 2019 to July 2019, using as a base, forms and / or files validated internationally and adapted to our reality, in which information was collected from the patient history, and a survey with 3 reagents of dichotomous responses. Results: The male sex was $56.5 \%$ and the age group of 20 to 29 years old was $32.7 \%$. According to the clinical classification: He showed complicated acute appendicitis 53.1\% (78 patients) and uncomplicated acute appendicitis $46.9 \%$ (69 patients). Regarding histopathological stages, $33.3 \%$ were phlegmonous or suppurated, gangrenous or necrotic and perforated with $26.5 \%$ both, and $13.6 \%$ congestive or catarrhal. The time between the onset of symptoms and admission to the hospital was less than 24 hours $51.7 \%$. Time between hospital admission and surgery reported more than 6 hours with $68 \%$. Self-medication was $65.3 \%, 59.9 \%$ did not receive prehospital medical care. In intra-abdominal complications, localized 
peritonitis and appendicular plastrón predominate with $50 \%$ and $12.5 \%$ respectively. Conclusions: Complicated acute appendicitis was associated with factors such as self-medication and the time elapsed between the onset of symptoms and surgical intervention.

Keywords: Complicated acute appendicitis and associated factors.

\section{INTRODUCCIÓN.}

La apendicitis aguda es el cuadro quirúrgico de urgencia más frecuente, y es la causa principal de abdomen agudo quirúrgico (1) siendo "la lesión inflamatoria del intestino de aparición muy frecuente y uno de los principales motivos de cirugía abdominal" (2). Estadísticas mundiales revisadas coinciden en indicar que "alrededor del 7-12\% de la población podría tener inminentemente un cuadro de apendicitis aguda en algún momento de su vida" $(3,4)$. Debido a su frecuencia, la enfermedad es tan impactante que se ha estimado con gran certeza que 1 de 15 individuos sufrirá de apendicitis en cualquier momento (3). Similar cifra reporta la Asociación Mexicana de Cirugía General, quien afirma que "la apendicetomía es en la actualidad el acto quirúrgico de urgencia con mayor frecuencia a nivel mundial, y que el riesgo aproximado de presentar apendicitis es de $8,6 \%$ para varones y de $6,7 \%$ en mujeres" (5). El apéndice es un tubo ciego y estrecho que se extiende desde la porción inferior del ciego y carece de una función útil conocida. La apendicitis suele ser provocada debido a una obstrucción que se origina en la luz del apéndice, por un fecalito (trozo endurecido de un material fecal) (2). Otros determinan que la apendicitis constituye una inflamación que se produce en el apéndice vermiforme, de forma generalmente aguda, que si no se diagnostica evolucionan rápidamente hacia complicaciones inminentes tales como la perforación y peritonitis (6). El síntoma más frecuente es un dolor constante que se localiza en el cuadrante inferior de la zona derecha del abdomen (específicamente en la fosa iliaca derecha), en torno al punto de Mc Burney y que comienza de forma intermitente en la región periumbilical (6). Es decir que el cuadro de la apendicitis aguda, evoluciona hacia diversos estadios, y empieza fundamentalmente con una etapa de tipo congestiva, y luego evolucionando a una apendicitis de tipo flemonosa, gangrenosa, y finalmente se presenta en forma perforada, produciéndose de esta manera que el contenido apendicular esté en contacto con la mayor parte de la cavidad abdominal (7).

Existen ciertos factores de origen local que ocasionan o producen la perforación temprana del apéndice cecal, tales como el tener trastornos de tipo circulatorio, y como consecuencia de una debilidad estructura del órgano (1). El tratamiento indicado de la apendicitis es únicamente de tipo quirúrgico (apendicetomía), siendo éste el procedimiento quirúrgico más frecuente en el mundo, las presentaciones atípicas son muy frecuentes durante los cuadros agudos, dentro de las que se destaca la variedad retrocecal (1). El retraso en la intervención por cualquier circunstancia, ya sea por el propio paciente que no acude inmediatamente a una institución especializada, automedicándose, o por motivos de retraso en el diagnóstico u otros factores, hace que "el cuadro evolucione a la perforación y peritonitis con liberación de materias fecales en la cavidad peritoneal" (6). En este sentido, haciendo una revisión bibliográfica al respecto, investigaciones realizadas en Cuba año 2004, reportan que, de un estudio de 214 casos, predominó el sexo masculino con 181 pacientes; 30,8\% tenían edades correspondientes a la 3era década, y que el diagnóstico anátomo-patológico supurada fue la cavidad más frecuente encontrada (8). El estudio en Loja, Ecuador año 2016 concluye que "los principales factores que ocasionan complicaciones en la apendicitis aguda son la automedicación y el tiempo transcurrido entre inicio de síntomas y la intervención quirúrgica (9). En Venezuela en un estudio realizado en el año 2013, concluye 
que es fundamental e importante que se identifique los principales factores de riesgo que ocasionan las complicaciones en la apendicitis aguda, lo que permitirá la disminución de la morbi-mortalidad de los pacientes (4). En lima, Perú año 2009 la causa más frecuente de abdomen agudo quirúrgico fue la apendicitis no complicada (10). En nuestro medio Ica, Perú, no es distante a esta realidad, observándose la concurrencia frecuente de operados con apendicitis aguda en su mayoría complicada, esto se debe al incremento de pacientes a nuestro nosocomio, por la migración, aunado a la referencia de pacientes de zonas aledañas.

Por todo lo narrado y analizado, consideramos de importancia, para la salud pública el abordar los factores asociados a apendicitis aguda que cursa con complicación, ya que, debido a un retraso en la atención de salud, la automedicación y el aliviar el dolor con la aplicación de remedios caseros por parte del paciente, agravarán la enfermedad pudiendo llegar a la muerte. $\mathrm{Y}$ es el profesional de enfermería y como parte del equipo multidisciplinario responsable de impartir programas educativos, enseñar a reconocer los signos y síntomas, lo que hay que hacer y especialmente lo que no hay que hacer cuando presenta dolor abdominal no determinado, referir inmediatamente a una institución y ser tratado por el especialista, conducente a disminuir las complicaciones por esta patología, lo que conlleva a una baja morbimortalidad, corta estancia hospitalaria, y reducción de costo económico para el paciente. Señalándose como objetivo identificar los factores asociados a la apendicitis aguda complicada en un hospital público de la ciudad de Ica.

\section{MATERIALES Y METODOS.}

La población estuvo conformada por todos los pacientes postoperados de apendicectomía en sus diversos estadios evolutivos del servicio de cirugía del Hospital Santa María del Socorro de Ica, Perú, haciendo un total de 980 pacientes postoperados que se obtuvo tomando como referencia datos históricos. La población de pacientes que fueron hospitalizados en los 5 años anteriores, obteniendo por promedio simple. Quedando la muestra constituida por los pacientes con el diagnóstico de apendicitis aguda e intervenidos quirúrgicamente, hospitalizados en el servicio de cirugía, obteniéndose prospectivamente en el periodo comprendido de diciembre 2018 a julio 2019, los cuales hicieron un total de 147 pacientes postoperados mayores de 14 años. El estudio hizo uso del método descriptivo, observacional, analítico, prospectivo de diseño no experimental de corte transversal. Integrada a una encuesta comprendida de 2 partes con 3 reactivos dicotómicos, y una ficha de recolección de datos el cual fue validado a nivel internacional $(4,9)$ aplicados en Venezuela y Ecuador, tomándose como base, con adaptaciones a nuestra realidad. Se empleó la historia clínica para el recojo de información. Se hizo uso del programa de análisis de datos SPSS versión 21, presentándose en tablas y gráficos. Para la prueba de hipótesis se aplicaron la prueba estadística de chi cuadrado $\left(\mathrm{X}^{2}\right)$.

\section{RESULTADOS.}

Se realizó con una muestra de 147 pacientes postoperados de apendicetomía con el diagnóstico clínico de apendicitis aguda en sus diferentes estadios evolutivos, predominó el sexo masculino (56,5\%) 83 participantes, grupo etario de $20-29$ años (32,7\%) 45 de ellos y procedían de áreas urbanas (77,6\%) 114 pacientes postoperados. Se clasificaron clínicamente de acuerdo a los estadios histopatológicos con apendicitis aguda complicada que mostró $(53,1 \%)$ con 78 pacientes, y apendicitis aguda no complicada $(46,9 \%)$ con 69 pacientes postoperados. Dentro de las apendicitis no complicadas se incluyeron a las apendicitis congestivas o catarrales con 13,6\% (20 pacientes) del total, y apendicitis flemonosas 0 supurativas $(33,3 \%)$ con 49 de los casos. Dentro del grupo de apendicitis complicada se consideraron a 
apendicitis gangrenosa 0 necrótica y apendicitis perforada (26,5\%) con 39 pacientes cada uno (Tabla 1). El tiempo transcurrido entre el inicio de los síntomas y la intervención quirúrgica se observó tiempo mayoritario de casos entre 48 horas a más de 72 horas con $72,8 \%$ (107 casos del total) correspondiente a apendicitis aguda complicada el mayor porcentaje con 40,8 \% (60 casos) (Tabla 2). En el análisis bifactorial entre clasificación clínica de apendicitis aguda y factores de riesgo, se halló asociación más frecuente en la apendicitis no complicada, y en tiempo menor de 24 horas para buscar atención hospitalaria (33,3\%) (49 del total) y no haber recibido atención médica prehospitalaria $(34,7 \%) 51$ participantes; en el grupo de apendicitis complicada las asociaciones frecuentes se determinaron entre estos y la automedicación (44,2\%) 65 pacientes (Tabla $3)$, haberse realizado intervención quirúrgica después de 6 horas de ingreso al hospital $(37,4 \%) 55$ pacientes . En el análisis bifactorial entre la clasificación clínica de la apendicitis aguda y las variables sociodemográficas, se halló asociación positiva frecuente entre apendicitis complicada y el sexo masculino $33,3 \%$ (49 de ellos), grupo etario de 20-29 años $17 \%$ (25 pacientes), y lugar de procedencia urbana $(40,8 \%)$ con 60 de los participantes, todas las asociaciones mencionadas no resultaron estadísticamente no significativas ( $p$-valor $>0,05)$ en la prueba de hipótesis aplicada (Tabla 4).

Tabla 1. Distribución porcentual de los encuestados según clasificación clínica de apendicitis aguda y estadios histopatológicos

\begin{tabular}{lc}
\hline \multicolumn{1}{c}{ Variable } & $\mathbf{N}=\mathbf{1 4 7}$ \\
\hline Clasificación clínica & \\
Complicada & 53,1 \\
No complicada & 46,9 \\
\hline Estadío histopatológico & \\
Apendicitis congestiva o catarral & 13,6 \\
Apendicitis flemonosa o supurativa & 33,3 \\
Apendicitis gangrenosa o necrótica & 26,5 \\
Apendicitis perforada & 26,5 \\
\hline Fuente: Base de datos &
\end{tabular}

Tabla 2. Distribución porcentual de los encuestados según clasificación clínica de apendicitis aguda y tiempo transcurrido entre el inicio de los síntomas y la intervención quirúrgica

\begin{tabular}{|c|c|c|c|c|c|c|c|}
\hline \multirow{4}{*}{ Variable } & \multicolumn{4}{|c|}{$\mathrm{N}=147$} & \multirow{3}{*}{\multicolumn{3}{|c|}{$\begin{array}{c}\text { Prueba de hipótesis } \\
\text { (Interpretación) }\end{array}$}} \\
\hline & \multicolumn{4}{|c|}{ Clasificación clínica } & & & \\
\hline & \multicolumn{2}{|c|}{ Complicadas } & \multicolumn{2}{|c|}{ No complicadas } & & & \\
\hline & \multicolumn{2}{|c|}{$n=78$} & \multicolumn{2}{|c|}{$n=69$} & & & \\
\hline Tiempo & $\mathrm{n}$ & $\%$ & $\mathrm{n}$ & $\%$ & $\mathrm{X}^{2}$ & $\mathrm{gl}$ & $\mathrm{p}$ valor \\
\hline Menos de 24 horas & 10 & 6,8 & 16 & 10,9 & & & \\
\hline De 24 a 48 horas & 8 & 5,4 & 6 & 4,1 & 8,44 & 3 & 0,038 \\
\hline De 48 a 72 horas & 29 & 19,7 & 12 & 8,2 & & nific & \\
\hline Más de 72 horas & 31 & 21,1 & 35 & 23,8 & & & \\
\hline
\end{tabular}

Fuente: Base de datos 
Tabla 3. Distribución porcentual de los encuestados según clasificación clínica de apendicitis aguda y factores de riesgo

\begin{tabular}{|c|c|c|c|c|c|c|c|}
\hline \multirow{3}{*}{ Variables. } & \multicolumn{4}{|c|}{$\begin{array}{c}\mathrm{N}=147 \\
\text { Clasificación clínica }\end{array}$} & \multicolumn{3}{|c|}{$\begin{array}{l}\text { Prueba de hipótesis } \\
\text { (Interpretación) }\end{array}$} \\
\hline & \multirow{2}{*}{\multicolumn{2}{|c|}{$\begin{array}{c}\begin{array}{c}\text { Complicada } \\
s\end{array} \\
n=78\end{array}$}} & \multicolumn{2}{|c|}{ No Complicadas } & \multirow[b]{3}{*}{$\mathrm{X}^{2}$} & \multirow[b]{3}{*}{ gl } & \multirow[b]{3}{*}{$p$ valor } \\
\hline & & & & & & & \\
\hline $\begin{array}{l}\text { Tiempo transcurrido entre el inicio de } \\
\text { los síntomas y el ingreso al hospital. }\end{array}$ & $\mathbf{N}$ & $\%$ & $\mathbf{n}$ & $\%$ & & & \\
\hline Menos de 24 horas & 27 & 18,4 & 49 & 33,3 & 21,57 & 3 & 0,000 \\
\hline De 24 a 48 horas & 25 & 17,0 & 13 & 8,8 & \multicolumn{3}{|c|}{ (Significativa) } \\
\hline De 49 a 72 horas & 12 & 8,2 & 5 & 3,4 & & & \\
\hline Más de 72 horas & 14 & 9,5 & 2 & 1,4 & & & \\
\hline $\begin{array}{l}\text { ¿Ud. para aliviar su dolor abdominal } \\
\text { tomó algún medicamento? }\end{array}$ & $\mathbf{N}$ & $\%$ & $n$ & $\%$ & $\mathrm{X}^{2}$ & gl & $\mathrm{p}$ valor \\
\hline Sí & 65 & 44,2 & 31 & 21,1 & 23,83 & 1 & 0,000 \\
\hline No & 13 & 8,8 & 38 & 25,9 & \multicolumn{3}{|c|}{ (Significativa) } \\
\hline $\begin{array}{c}\text { ¿Usted recibió atención médica pre } \\
\text { hospitalaria? }\end{array}$ & $\mathbf{N}$ & $\%$ & $n$ & $\%$ & $\mathrm{X}^{2}$ & gl & p valor \\
\hline Sí & 41 & 27,9 & 18 & 12,2 & 10,68 & 1 & 0,01 \\
\hline No & 37 & 25,2 & 51 & 34,7 & \multicolumn{3}{|c|}{ (Significativa) } \\
\hline
\end{tabular}

Fuente: Base de datos

Tabla 4. Distribución porcentual de los encuestados según la clasificación clínica de apendicitis aguda y características sociodemográficas

\begin{tabular}{|c|c|c|c|c|c|c|c|}
\hline \multirow{4}{*}{ Variables. } & \multicolumn{4}{|c|}{$N=147$} & \multirow{4}{*}{\multicolumn{3}{|c|}{$\begin{array}{l}\text { Prueba de hipótesis } \\
\text { (Interpretación) }\end{array}$}} \\
\hline & \multicolumn{4}{|c|}{ Clasificación clínica } & & & \\
\hline & \multirow{2}{*}{\multicolumn{2}{|c|}{$\begin{array}{c}\text { Complicadas } \\
n=78\end{array}$}} & \multirow{2}{*}{\multicolumn{2}{|c|}{$\begin{array}{l}\text { No complicadas. } \\
\qquad n=69\end{array}$}} & & & \\
\hline & & & & & & & \\
\hline Sexo & $\mathbf{n}$ & $\%$ & $\mathbf{n}$ & $\%$ & $\mathrm{X}^{2}$ & gl & p valor \\
\hline Femenino & 29 & 19,7 & 35 & 23,8 & 2,73 & 1 & 0,098 \\
\hline Masculino & 49 & 33,3 & 34 & 23,1 & \multicolumn{3}{|c|}{ (No significativo) } \\
\hline $\begin{array}{l}\text { Grupos } \\
\text { etarios }\end{array}$ & $\mathbf{n}$ & $\%$ & $\mathbf{n}$ & $\%$ & $X^{2}$ & gl & p valor \\
\hline 10 a 19 & 8 & 5,4 & 15 & 10,2 & 10,11 & 5 & 0,072 \\
\hline 20 a 29 & 25 & 17,0 & 23 & 15,6 & \multicolumn{3}{|c|}{ (No significativo) } \\
\hline 30 a 39 & 19 & 12,9 & 12 & 8,2 & & & \\
\hline 40 a 49 & 7 & 4,8 & 10 & 6,8 & & & \\
\hline 50 a 59 & 8 & 5,4 & 7 & 4,8 & & & \\
\hline$\geq 60$ & 11 & 7,5 & 2 & 1,4 & & & \\
\hline $\begin{array}{c}\text { Lugar de } \\
\text { procedencia }\end{array}$ & $\mathbf{n}$ & $\%$ & $\mathbf{n}$ & $\%$ & $\mathrm{X}^{2}$ & gl & $p$ valor \\
\hline Urbano & 60 & 40,8 & 54 & 36,7 & 0,038 & 1 & 0,846 \\
\hline Rural & 18 & 12,2 & 15 & 10,2 & \multicolumn{3}{|c|}{ (No significativo) } \\
\hline
\end{tabular}

Fuente: Base de datos 


\section{DISCUSIÓN.}

Se reporta $53,1 \%$ de casos de apendicitis aguda complicada, coincidiendo con el estudio de Álvarez año 2014, quien registra "el 58,60\% de casos fueron formas complicadas" (11).

En cuanto a los hallazgos respecto al sexo y edad, el estudio mostró predominio del sexo masculino $56,5 \%$ y el grupo etario de $20-29$ años con $32,7 \%$ fue el mayoritario, coincidente estos resultados con estudios revisados como el de: Ferrer et al en Cuba, quien señala que, de 214 casos, 181 fueron de sexo masculino, $30,8 \%$ estaba en la tercera década de la vida (8). Velásquez et al en México, cuyo hallazgo fue $57,1 \%$ evidenció en mayor proporción el sexo masculino, y la edad media fue de 29,44 (12). Gamero en Lima, Perú reporta que, del total de pacientes operados por apendicitis aguda, el $60 \%$ fueron hombres con edad promedio de 31 años (10), y Álvarez en Arequipa, Perú, señalaron que el $64,33 \%$ de pacientes fueron varones y el $28,05 \%$ de pacientes con apendicitis tuvieron de 21 a 30 años (11). Sin embargo, no fue consistente con el sexo en estudios de: Huacuja et al en México (13), con García et al en Ecuador (14) y con el estudio de Arcana en Lima, Perú (15), en donde predominó el sexo femenino con porcentajes $51,5 \%, \quad 61,54 \%$ y $57,6 \%$ respectivamente. Como se puede apreciar, el estudio realizado y en otras revisadas, en su gran mayoría destacaron predominio porcentual del sexo masculino y la edad fueron los determinantes en riesgo que evidenciaron estos casos, consistente con lo referido con la literatura que señala que "la apendicitis aguda se presenta con mayor frecuencia en adolescentes y adultos jóvenes, sobre todo en hombres" (2). "La misma que puede presentarse en cualquier edad, su incidencia aumenta durante la infancia y alcanza un pico entre los 10 y 30 años de edad, declinando a partir de la cuarta década" (3).

Referente a los estadios histopatológicos o anatomopatológicos, el estudio mostró el mayor hallazgo en intraoperatorio fue la apendicitis flemonosa o supurativa $33,3 \%$, siendo estos porcentajes concordantes o similares con los estudios de: Gamero (10), Amundaray (4), Ferrer (8) Álvarez (11), aunque en mayor porcentaje, comparativamente con el estudio, con $40 \%$, $50 \%$, $64,5 \%$ y $93,85 \%$ respectivamente fueron diagnosticados como apendicitis supurada o flemonosa. Este estadio de apendicitis se distingue por mostrar "la serosa intensamente congestiva, edematosa, coloración rojiza y con exudado fibrino - purulento en su superficie, si bien aún no hay perforación de la pared apendicular, se produce difusión del contenido mucopurulento introluminal hacia la cavidad libre" (16).

En relación a los factores de riesgo (tiempo transcurrido entre el inicio de los síntomas y el ingreso al hospital) con la clasificación clínica de la apendicitis aguda, se observó la no complicada el mayor porcentaje $33,3 \%$ fue el tiempo menor de 24 horas para buscar atención médica hospitalaria luego de iniciado los síntomas y un lapso de tiempo de evolución mayor reportado para apendicitis aguda complicada. Estos resultados son concordantes con otros autores: Amundaray, quien registró que el $42 \%$ de los pacientes fueron diagnosticadas en menos de 24 horas (4), Álvarez reporta que la media de tiempo transcurrido desde el inicio de los síntomas hasta la atención en emergencia fue de 18 horas en la apendicitis aguda no complicada, que la complicada el tiempo fue mayor (11). El tiempo de evolución de la enfermedad es un factor de riesgo causal, evidenciado en el muestreo de casos estudiados prospectivamente, y estos están en relación con la rápida atención médica, el cual reduce la progresión de los síntomas y su consecuente complicación que sin duda está asociado a otros factores. El estudio determinó asociaciones frecuentes de la apendicitis aguda complicada, con la automedicación $44,2 \%$, haber realizado la intervención quirúrgica después de 6 horas de su ingreso al hospital $37,4 \%$ y un tiempo de hospitalización 
mayor a 6 días con 39,5\%. Siendo estos resultados consistentes con otros autores, aunque en mayor porcentaje al estudio efectuado, Álvarez registró $70,97 \%$ se demoró más de 12 horas para la realización de la intervención quirúrgica, y 70,9\% recibieron medicación previa en apendicitis aguda complicada (11). Angamarca reportó que se automedican $62,12 \%$, difiriendo ligeramente con el estudio en cuanto al tiempo de espera para cirugía que fue 3-4 horas (33,33\%) (9).

El tiempo de espera para la realización de la cirugía que se prolonga por una serie de exámenes auxiliares que se realiza para la determinación definitiva del diagnóstico y por otras circunstancias propias institucionales, aunada a la automedicación previa que enmascaran el cuadro y su consecuente progresión y agravamiento de los estadios evolutivos, desencadenando la presentación características de la apendicitis aguda complicada, brechas reportadas de estos factores asociados que se tendrá que intervenir para acortar el tiempo de espera y evitar la automedicación, a través del despliegue de programas educativos acerca de la apendicitis aguda y sus consecuencias y/o complicaciones conducentes a la disminución de la misma.

\section{El tiempo transcurrido entre el inicio de los} síntomas y la intervención quirúrgica, se observó en el estudio un tiempo mayoritario de casos entre 48 horas a más de 72 horas con $72,8 \%$ (107 casos en total), correspondiéndole a apendicitis aguda complicada el mayor porcentaje $40,8 \%$ (60 casos), a comparación de la apendicitis aguda no complicada con $32 \%$ (47 casos), lo que explica que cuanto más tiempo se extiende para la realización de la intervención quirúrgica, evoluciona progresivamente los estadios histopatológicos, desencadenando las consecuentes complicaciones que mostró el estudio. Esto concuerda con Álvarez quien mostró que la media del tiempo transcurrido desde el inicio de los síntomas hasta la cirugía fue de 41,7 horas en el grupo de la apendicitis complicada, y de 26,7 horas en el de la apendicitis no complicada (11). Asimismo, concuerdan con Álvarez, quien mostró que la media del tiempo transcurrido desde el inicio de los síntomas hasta la cirugía fue de 41,7 horas en el grupo de la apendicitis complicada y de 26,7 horas en el de la apendicitis no complicada. Se encontró diferencia significativa $(p<0.05)(11)$.

En cuanto a las complicaciones se hallaron 78 casos $(53,1 \%)$ de un total de 147 pacientes, correspondiendo para apendicitis gangrenada o necrótica y perforada $26,5 \%$ cada una; estos a su vez 40 casos $(51,3 \%)$ presentaron complicaciones intraabdominales de los cuales fueron peritonitis localizada 50\% (20 casos), generalizada $7,5 \%$ ( 3 casos) y plastrón apendicular 42,5\% (17 casos) e infección de la herida operatoria con $5,1 \%$ (4 casos), de los cuales 2 casos estuvieron asociados a la presencia de un plastrón apendicular en la fase aguda de la enfermedad. Estos resultados concuerdan con autores: Medina registró $49,8 \%$ apendicitis gangrenada, perforada $18,9 \%$, peritonitis localizada $12,3 \%$, plastrón apendicular $6,6 \%$ y peritonitis generalizada $9,3 \%$ con variación debido a un mayor muestreo (227 casos en total) (17). Angamarca halló casos de perforación $31,81 \%$, peritonitis $27,27 \%$, plastrón apendicular $36,36 \%$, y 1 caso de infección de herida operatoria (9). Complicaciones significativas que se desarrollaron en la apendicitis aguda que se revelan en el estudio, como en otros analizados, que está relacionada a factores asociados como demora en la atención médica e intervención quirúrgica, automedicación, entre otros como el sistema inmunológico sui generis del paciente, que propicia la evolución de la enfermedad.

\section{CONCLUSIONES.}

La apendicitis aguda complicada se asoció a factores como la automedicación y el tiempo transcurrido entre el inicio de los síntomas y la intervención quirúrgica. 


\section{CORRESPONDENCIA.}

Dra. Isabel Natividad Urure Velazco

Correo electrónico: isabelnauv@yahoo.com

\section{REFERENCIAS BIBLIOGRÁFICAS.}

1. De Elorza G. Manual de Enfermería Zamora. Colombia; 2007: p. 237.

2. Swearingen P. Enfermería Médico Quirúrgica. 6ำ ed. Barcelona, España: Ed. Elsevier; 2012: Capítulo 7, p. 633.

3. Ministerio de Salud Pública. Protocolos de atención para cirugía. 1ำed. Volumen I. Santo Domingo; 2016.

4. Amundaray A, Fleitas P. Factores de riesgo asociado a complicaciones en pacientes con apendicitis aguda. Tesis especialidad. Caracas, Venezuela. Facultad de Medicina, Universidad Central de Venezuela; 2013.

5. Gonzáles JR, et al. Guía práctica clínica Apendicitis Aguda. Asociación Mexicana de Cirugía General AC. México D.F; 2014.

6. Océano Mosby. Diccionario de Medicina. Barcelona, España: Ed. Océano; 1997.

7. Mihura M. La apendicitis aguda es la causa más frecuente de cirugía abdominal de Urgencia. Diario Argentino INFOBAE. 16 Agosto; 2018.

8. Ferrer H, Ferrer H, Mesa O, Gazquez S. Apendicitis aguda: Estudio estadístico de 214 casos. La Habana, Cuba. Rev. Cubana Invest. BIOMED. 2007; 26(3).

9. Angamarca E. Factores asociados a la apendicitis aguda complicada en los adultos hospitalizados en el área de cirugía del Hospital Regional Isidro Ayora de Loja en el periodo Julio-Diciembre 2015. Tesis pregrado. Loja, Ecuador. Área de Salud Humana, Carrera de Medicina Humana, Universidad de Loja; 2016.
10. Gamero M, Barreda J, Hinostroza G. Apendicitis aguda: Incidencia y factores asociados. Hospital Nacional "Dos de Mayo" Lima, Perú 2009. Tesis investigadores. Lima, Perú. Facultad de Medicina. Universidad Privada San Martín de Porres; 2009.

11. Álvarez JA. Factores de riesgo asociados a apendicitis aguda complicada en el Hospital Apoyo Camaná en los años 20122013. Tesis pregrado. Arequipa, Perú. Facultad de Medicina, Universidad Nacional de San Agustín de Arequipa; 2014.

12. Velásquez JD, Ramírez $F$, Vega J. Premedicación, factores de retraso en el diagnóstico y tratamiento quirúrgico de la apendicitis aguda. Guanajuato, Salamanca, México. Rev. Cirugía General 2009; 31(2): 105-109

13. Huacuja R, Ruiz M, Lemus R, Villegas $E$, Gonzáles M, Díaz A, et al. Factores predictores para apéndice blanca y apendicitis aguda en pacientes sometidos a apendicetomía. Experiencia de dos años en una institución privada. Rev Invest Med Sur Mex. 2015; 22 (1): 11-18

14. García AB, Díaz $X$, Chiroboga GL. Factores que retrasan el diagnóstico y tratamiento de la apendicitis aguda, Hospital Homero Castanier Crespo, Azogues 2013. Tesis pregrado. Cuenca, Ecuador. Facultad de Ciencias Médicas, Escuela de Medicina, Universidad de Cuenca; 2013

15. Arcana $\mathbf{H}$. Factores relacionados con la apendicitis aguda complicada en pacientes de 15 a 60 años en emergencia del HNAL un enfoque basado en la evidencia 2003. Tesis postgrado (especialista). Lima, Perú. Facultad de Medicina, Unidad de Postgrado, Universidad Nacional Mayor de San Marcos; 2004. 
16. Wong P, Morón P, Espino C, Arévalo J, Villaseca R. Apendicitis aguda (Internet). Lima, Perú. Universidad Nacional Mayor de San Marcos. (Citado 24 de Agosto 2018). Disponible en: http://sisbib.unmsm.edu .pe/bibvirtual/libros/medicina/cirugia/tomo i/Cap 11 Apendicitis\%20aguda.htm
17. Medina 0 . factores asociados a la apendicitis aguda complicada en el Hospital de Apoyo II-2 Sullana-Piura Enero-Diciembre 2014. Tesis pregrado. Sullana, Piura, Perú. Facultad de Medicina Humana, Universidad Nacional de Piura; 2015.

Recibido: 10/11/2019

Aprobado para publicación: 10/03/2020 